\title{
Arabic Proverbs and Challenges in Translating them into English
}

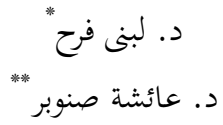

ISSN (P) 2664-0031 (E) 2664-0023

DOI: https://doi.org/10.37605/fahmiislam.v4i1.232

Received: March 19,2021

Accepted: March 22, 2021

Published: June 30,2021

\section{Abstract}

Proverbs and fixed expression are the mirror which reflects people's nature and the resume of what they have experienced. Proverbs are part of metaphorical language that has superficial as well as a deep sense. They are form of speech or an expression, it can't understand by individual meaning. Role of culture is important in target language.

These proverbs have been written briefly in small sentences to narrate long stories that are the main bey to understand human culture and social reality. Therefore, translating proverbs are more difficult for the translator, who are not familiar with them. The variations in cultures make translation process real challenge.

Every proverb there are links and characters associated with it, where the translator had to look for the synonym identical to proverb similar in meaning and composition.

Keywords: Idioms, translation, difficulties, strategies.

تقرب الأمثال المراد بالعقل للقلب، وتثبت المعنى في النفس لحسن التشبيه بها، لأن لها

لوناً بديعياً لا مثيل لها في نظائرها من مجالات الأدب، وهي كعقد اللؤلؤ على جيد حسناء، وهي

تحمل في ثناياها تراثاً فكرياً، وثقافياً، و اجتماعياً. الأمثال لها مكانة خاصة في جميع الشعوب،

حيث تشترك أغلب لعناصر اللغوية بين لغات العالم. وكانت الأمثال في الثثر الجاهلي منتشرة لذا

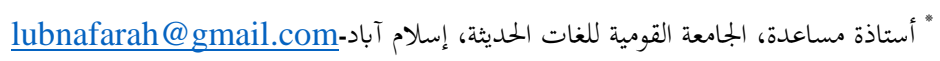

"استاذة مساعدة، قسم الدراسات الإسلامية، جامعة النساء سردار بهادر خان، كويتا- 
استخدمها القرآن الكريم لتوضيح المعنى وإبراز أهميته; لأن الحياة الأنسانية تحتاج لإيضاح المعنى عبر

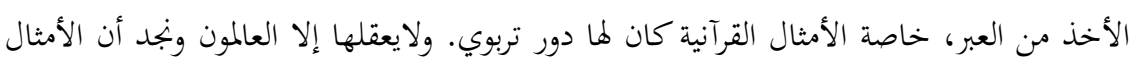

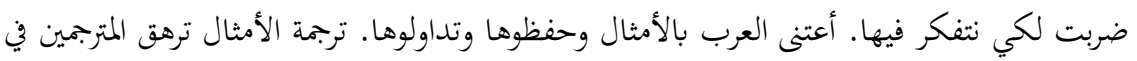

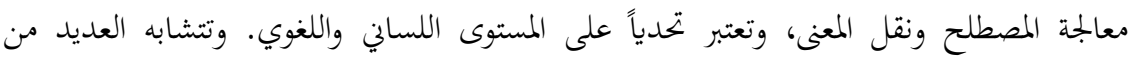

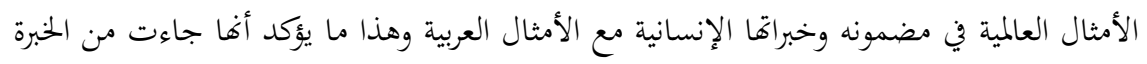
الإنسانية وسنتبع منهج استنباطي تحليلي في استخراج المعنى من الأمثال. وربط ترجمتها

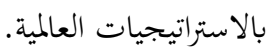

الدراسة سوف تسلط الضوء على استراتيجيات يحتذيها المتربمون عند ترجمة الأمثال بما

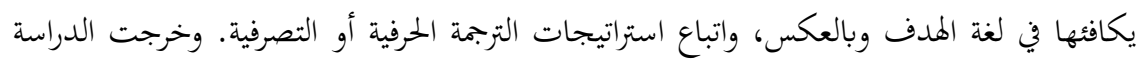
بعدد من التوصيات ستساهم في ضبط التربمة وتوحيدها في الحقول اللسانية. الكلمات الداله: الأمثال، الإيجاز، الترجمة، الاستراتيجيات.

المقدمة

لقد نشأت فكرة البحث من خلال ممارستي لعملي في حقل الترجمة الأدبية، والصعوبات

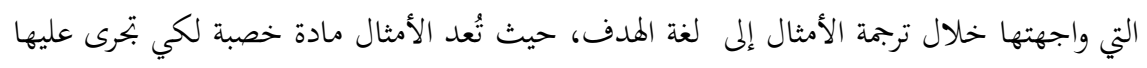

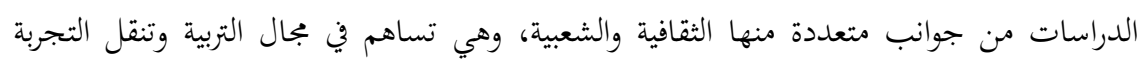
للمتلقي، لكي يجد فيها الأصالة والصدق. فيما يتعلق بالترجمة بين الثقافات، هناك دائمًا فجوة، ومن الصعب سد هذه الفجوة خاصة عند ترجمة التعابير من لغة إلى أخرى. وهذا يؤدي إلى العديد من المشاكل في عملية فهم الأمثال ومن ثم ترجمتها.

والأمثال تكون مرآة للشعوب وتنبع من بتاربهم، فتكون مصوغة في جمل قصيرة وهي

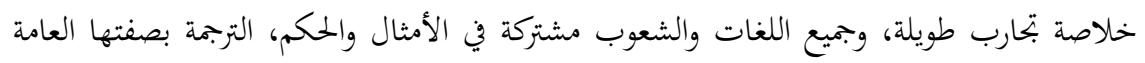
تكون "خيانة" بسبب صعوبة إيجاد مرادفات، لذا تشكل ترجمة الأمثال أكثر صعوبة للمترجم، لأن 
المثل يكون وراءه خيوط وخصائص ترتبط به فتحتم على المترجم أن ييحث المرادف المتطابق له في المعنى والتركيب.

الأمثال تكون الأرقى في الفن الأدبي حيث لها أثر عميق على الأنسان وخاصة أهما تشترك في عناصرها بين جميع اللغات، وهي تحمل دلالات حضارية عندما نسعى لتربمة الأمثال فإننا

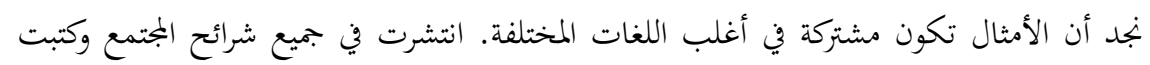
العديد من الأبحاث حول الأمثال حيث اعتنى بعض الباحثين على دراستها من الناحية اللغوية

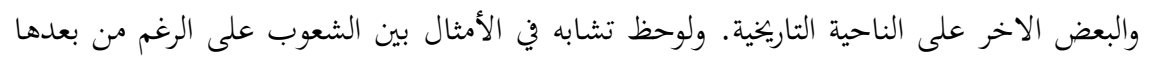

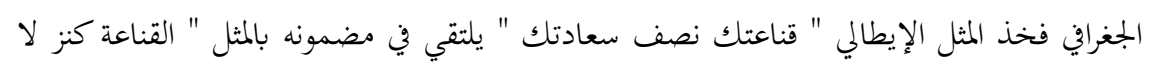
يفني تعتبر ترجمة الأمثال تحدياً كبيرا لأفما تتطلب ثقافة ومعرفة لغوية حيث على المترجم أن

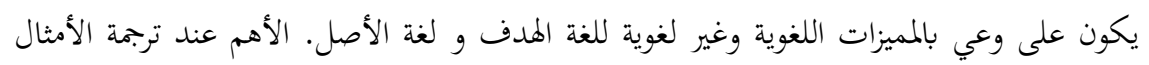

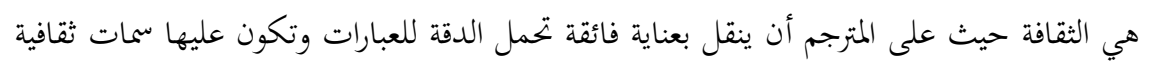

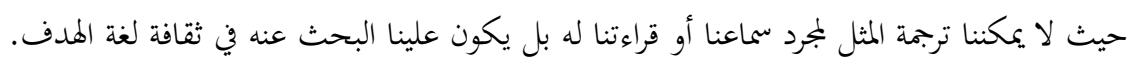

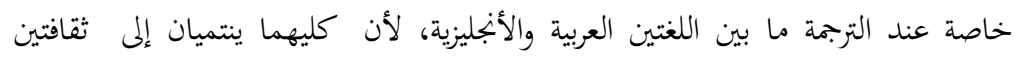

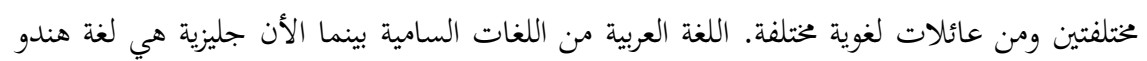

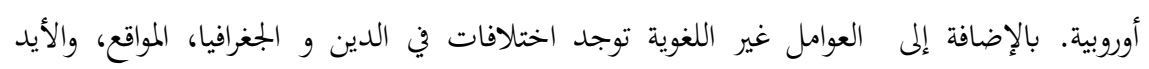
يولوجيات المختلفة مما يصعب عملية الفهم ومن ثم ترجمة الاصطلاحات.

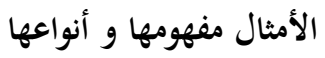

$$
\begin{aligned}
& \text { حسب شرح لسان العرب لابن منظور: } \\
& \text { "المشاهة، مثلهُ أي شبههُ" }
\end{aligned}
$$

المثل: هو قول انتشر في أفاق الشعب. و يتميز بخصائص لكي ينضم للأجناس الأدبية. يكون خالياً

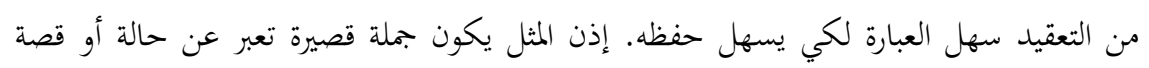


تضمن موعظة بلغة جميلة وعبارة أنيقة1. إبن خلدون يعرفها بأهما منوال هي القالب الذي تفرغ فيه

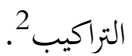

سمي المثل مثلاً لأنه يضرب به في العمل النظير الشبيه به. التمثال يكون صورة للشخص وهو يصور الأنسان لذا يسمى تمثالاً، والمثل لون أدبي أنتشر مثله مثل سائر الألوان الأدبية.

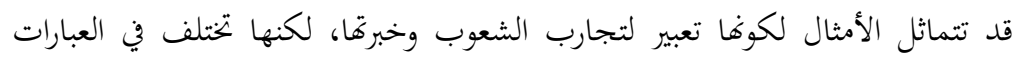

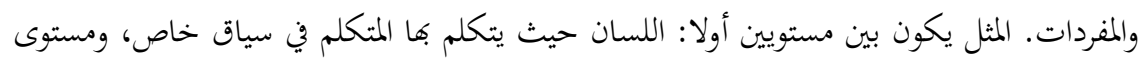
الكلام لكوفا مصطلحات فهنا تأتي مهمة المترجم حيث يبحث عن معادل لساني و مصطلح معادل ليترمها إلى لغة الهدف فتكون مهام المترجم إيجاد معادلات دلالية لفظية، ومرجعية وظيفية. والأمثال تنتمي إلى الفن، وفي النفس الوقت تنتمي إلى لغة الحديث اليومي، وتكون في الثنر والشعر، إلهاء وهذا يعني تواجدها في العملية اللغوية. على سبيل المثال، فإن المصطلح "conceited as a barber's cat" يضرب به مثلاً للشخص الذي لديه النشوة أو المبالغة، لا يمكن ترجمته ب" مغرور كقطة الحلاق " فالترجمة الحرفية

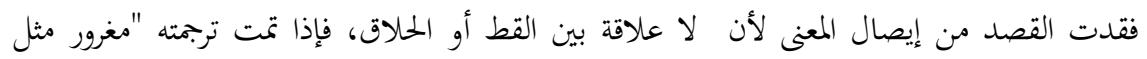

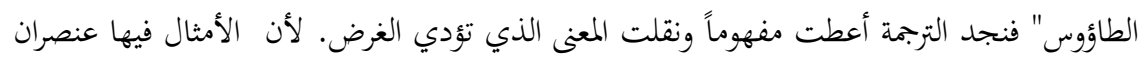

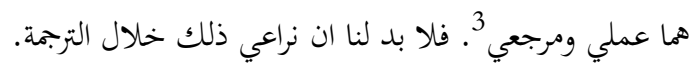

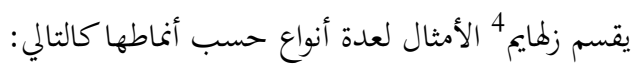

المثل: ما جاء من خبرات الحياة عبر تكرار من الأجيال ويكمن في داخله التجارب التي مر بها الشعب، ومنها قولم: عشب ولا بعير. التعبير المثلى: فهو يبرز أحوال المتكررة والعلاقات الإنسانية في جملة، سهلة نثرية مثال على ذلك:

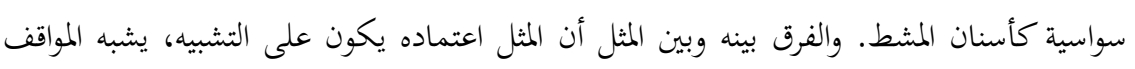

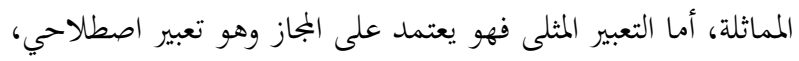

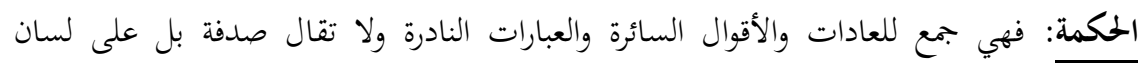

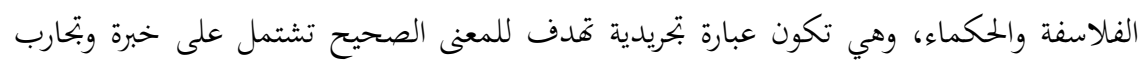
الحياة هدفها الموعظة وإسداء النصحّ. ومنها أن يضرب لسانك عنقك. 
العبارة التقليدية: عبارات تقال في التحية والدعاء واللعن، وتتوفر في كتب الأمثال مع أنه ليس من

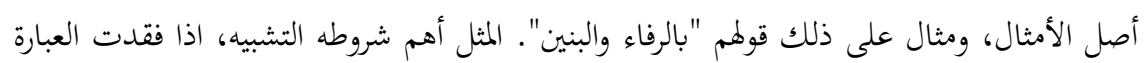
التشبيه فلا تكون مثلاً.

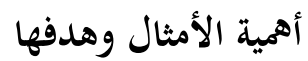

للأمثال أهمية من الناحية اللغوية، لأها تتميز بالدقة، وهي ثرية في ألفاظها وتدل على للى حس لغوي للشعوب التي تتداول عنها، ولاتخلو الحضارات القديمة والحديثة والثقافات من الأمثال.

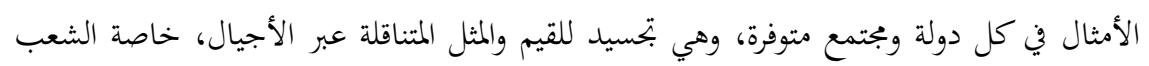

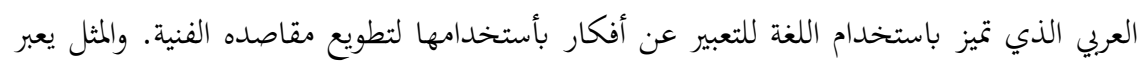

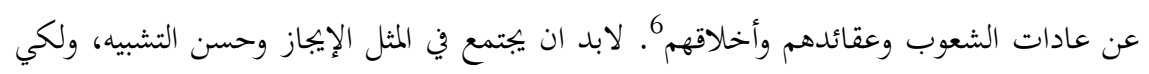

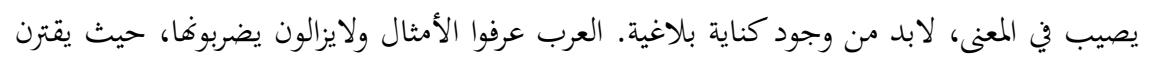

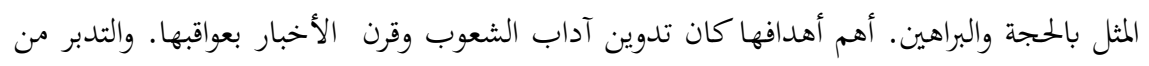

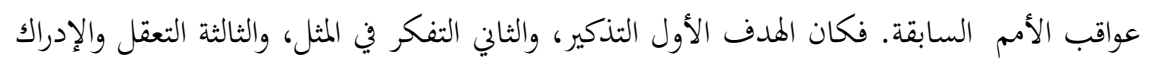

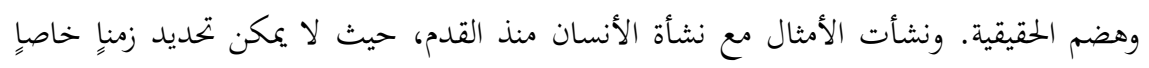

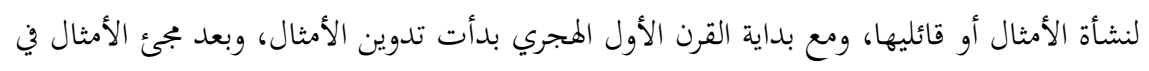

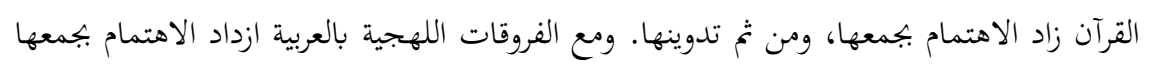

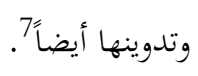

\section{خصوصية والبنية التركيبة للأمثال}

الأمثال دائما تتمتع بعفوية فهي تستخدم لغة وسمة عامة وعدم معرفة القائل الذي بدأها.

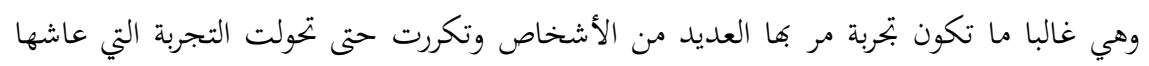
بحلاوقما ومرارةا لتكون مثلاً للأجيال القادمة وهي حكمة العرب في في الجاهلية والإسلام. المثل يكون سهلا لحفظه وكذلك بلاغي وعبارته وجيزة وجميلة التشبيه، وموجزة تشمل

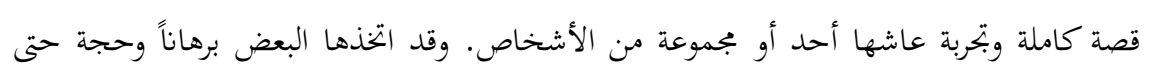

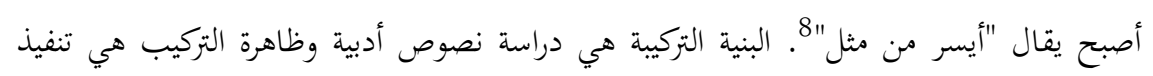


الكلام وسياق الخطاب ?. حيث نجد في الأمثال أنواع البناء المختلف فنجد مرة يأتي بأسلوب في

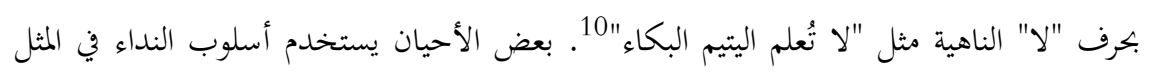

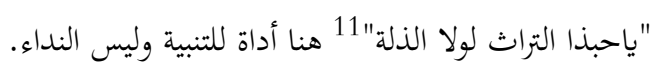

أسلوب النفي يستخدم لصرف ذهن سامعه من عمل الفعل بطريقة مباشرة وغير مباشرة.

$$
\text { ففي المثل "لن يهلك امرئ عرف قدره" }
$$

تشابه الأمثال عالميا

عندما نتابع الأمثال العالمية نجدها متشابه من ناحية المضمون والتجربة الإنسانية التي

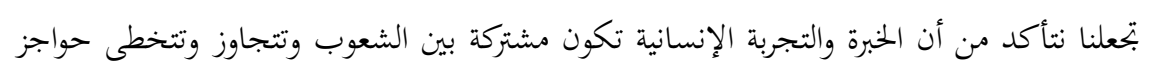
المكان.

العديد من الأمثال خرجت من البيئات الشرقية إلى الغربية والعكس تحمل محتوى إنساني

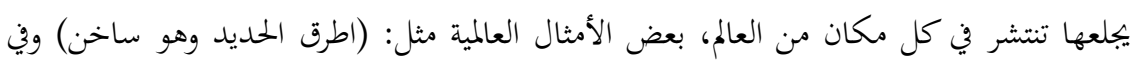

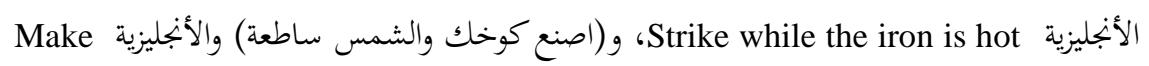
hيy while the sun shines

وكذلك الأمر في الأمثال السويسرية تتشابه مع الأمثال العربية رغم اختلاف في الثقافة

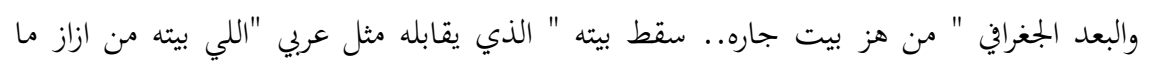

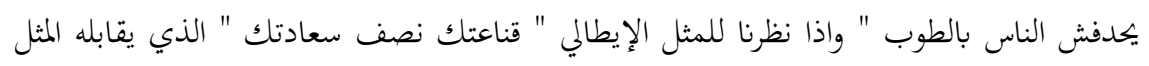

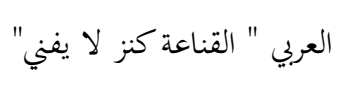

عندما نجد الفرنسيين يقولون المثل "إذا أردت أن تعرف رقي أمة فانظر إلى نسائها" فعند

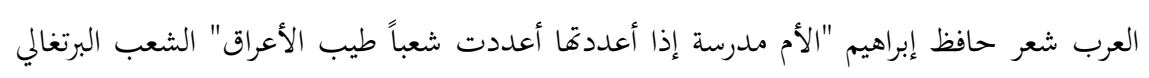

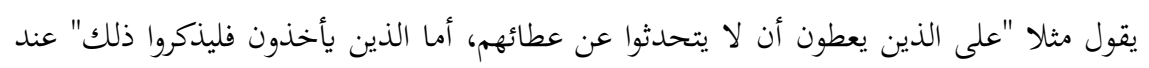

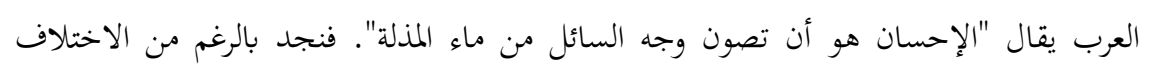
الجغرافي والبعد المكاني والثقافي بين الشعوب لكن الأمثال تتشابه. 
فالمثل الفرنسي يقابلها أمثولة للشاعر حافظ ابراهيم " "، والمثل الأمريكي " كثيرا ما نرى

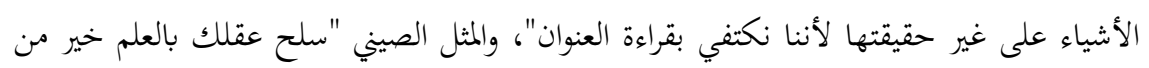
أن تزين جسدك بالجواهر"، والمثل الروسي أخطاء الأخرين دائما أكثر لمعانا من أخطائنا، والمثل

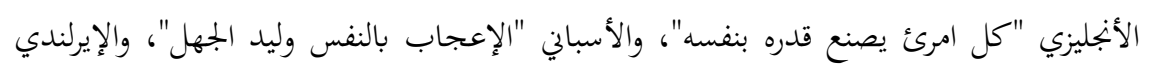

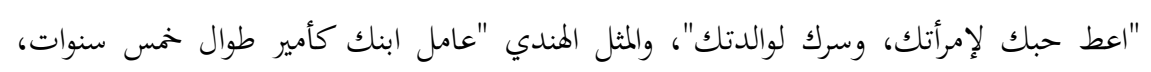

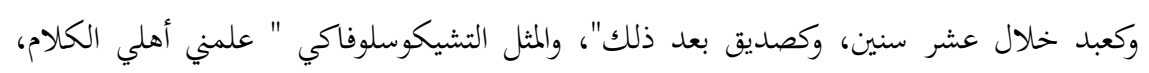

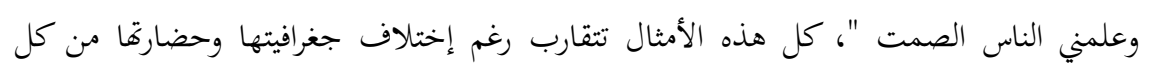
شخص لآخر على أرض البسيطة.

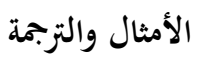

تعتبر الترجمة تحديا لكنها تكون تحديأ كبيراً إذا ما كانت ترجمة ثقافية وبالخصوص ترجمة

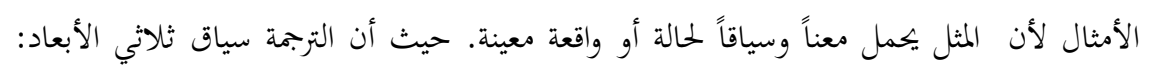

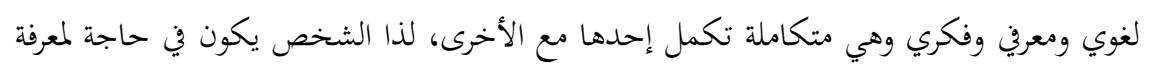

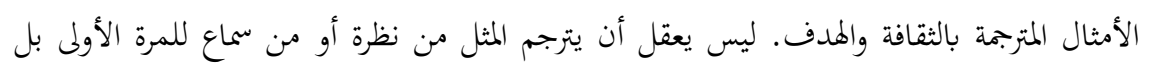
يمتاج إلى التعمق في كلماته وأصله. الترجمة لها دور مهم في تبادل الثقافات وهي عملية نقل للنص بكامل سماته البلاغية

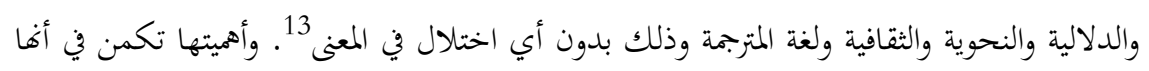
تأتي مصطلحات جديدة في حقول العلم لأفها تنقل المفاهيم عبر شرح الدلالات وإدراج المدلولات. ولقد اتفق العلماء على ضرورة وجود صلاحيات وضوابط خاصة عند مترجم المثل وهي:

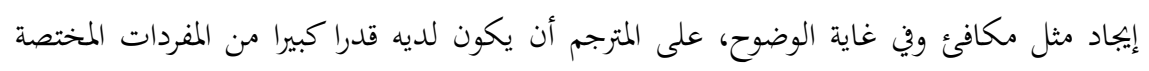

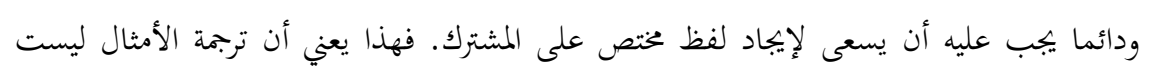
بالأمر الهين، بل تحتاج لتوافر شروط خاصة بالمترجم.

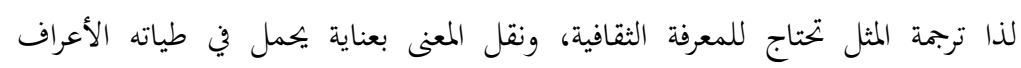
والسمات الثقافية الموجودة في المثل الذي نترجمه ولايمكنا ترجمة المثل خلال النظرة الأولى للكلمات لمعند 
بالمعجم، بل يحتاج للمعرفة العميقة التي يحتويها المثل 14. لأنه يجد نفسه في بعض الأحيان أمام نص

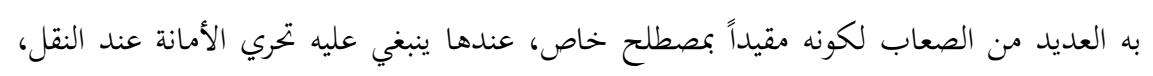

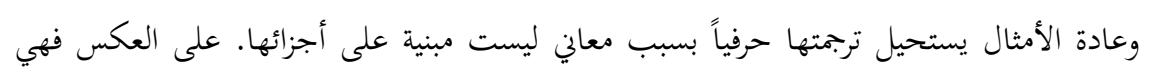

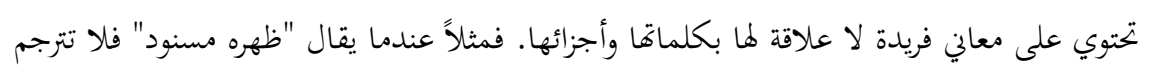

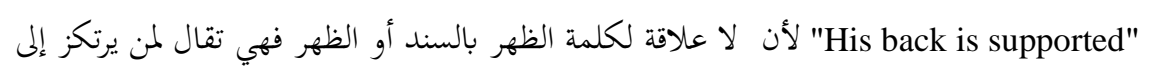
دعم مالي أو بشري قوي فترجتها "He is backed up".

\section{ترجمة المصطلحات والأمثال مفهوماً}

الترجمة هي نقل المعنى من لغة الأصل إلى لغة الهدف مع المحافظة على التكافؤ 15 ـوتمتاز

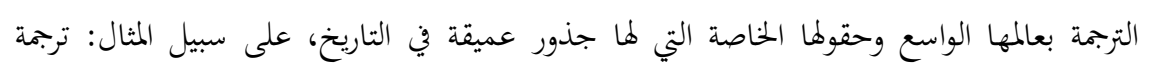

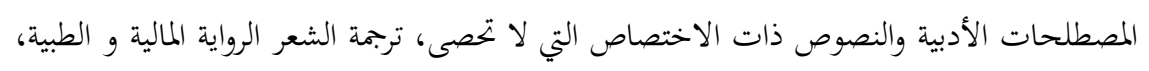
الصيدلية.... (لخ.

الترجمة ليست وليدة هذا العصر أو العصر الماضي بل عرفها العالم منذ تلاحقات

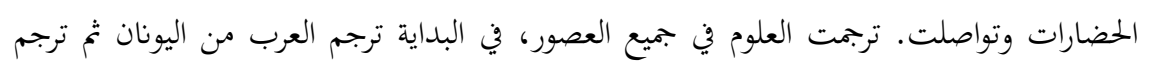
الأوروبيون إنجازات العرب في الطب والفلك. ترجن.

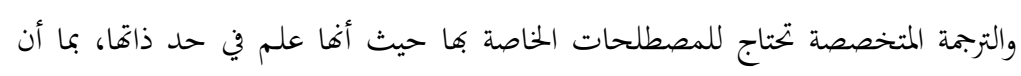

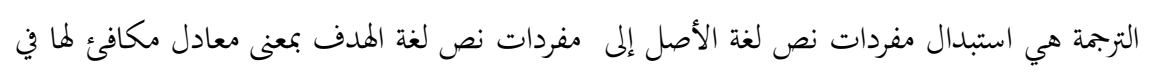

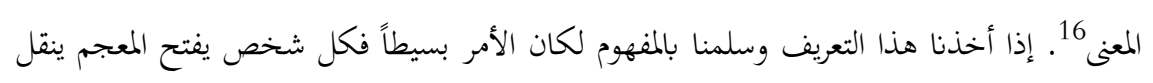

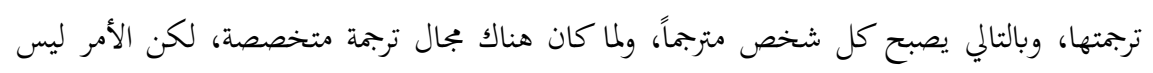

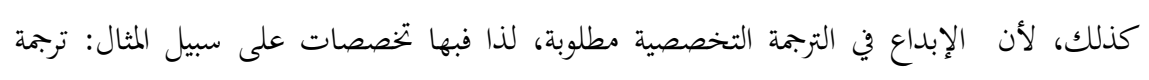

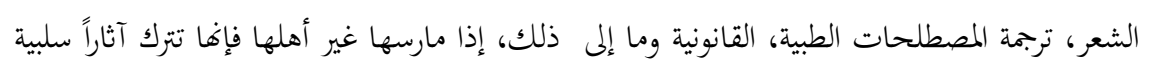

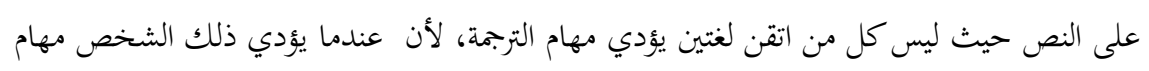
الترجمة بدون الخبرة الكافيه ينشأ عنه أخطاء صرفية ونوية ولغوية تكون عواقبها وخيمة على الأمة 
فإذن ترجمة المصطلحات والترجمة عموماً فن وعلم بها تخصصات ومجالات مثله مثل العلوم

الأخرى. على المترجم مواكبة تطورات المصطلحات في مجال تخصصه، لكي يمكنه إضفاء شكل جمالي على النصوص التي يترجمها، مع المحافظة على الدقة والمعنى والأمانة في نقل المفهوم 18. وشروط مترجم المصطلحات الخاصة لابد أن يتقن اللغتين المترجم منها والمترجم إليها، والمترجم لابد من توافر الصلاحية التامة لديه، حتى في بعض الأحيان يلجأ إلى وضع مصطلحات وبذا يكون مساهما في ترويج مفاهيم ومصطلحات مستجدة.19.

تتضح مشكلة ترجمة المصطلحات في الندوات واللقاءات السياسية فلكل مؤتمر أو لقاء شخصية سياسية توجد مصطلحات وعبارات خاصة تحتاج لإيجاد مكافئ لها لإيصال المعنى، يحتاج في بعض الأوقات إتباع المنهج المتزامن، المنهج التطوري، والمتعاصر. لكي لا يعطي معناً متلابساً ويعسر الجدل بين الفريقين خلال الاجتماع أو المؤتمر 20. العناصر الأساسية في ترجمة الأمثال العناصر الرئيسية في ترجمة الأمثال والتي تشكل صعوبات رئيسية في الترجمة هي: الدلالي: المعنى العام للمثل، والصورة التي تنشأ في ذهن السامع، لأن مفرداته قليلة ومعناه عميق. والمصطلح ممكن يكون تعبير ثابت خاص بعنصر ثقافي. لأن المعنى الدلالي لا يفهم للمترجم من أول مرة.

اللفظي: المحسنات اللفظية، والبلاغية والأساليب النحوية والتشبيه والكناية التي تلعب دوراً بارزاً في المثل ويهملها المترجم عند البحث عن مقابل لها. المرجعي: أماكن جغرافية أسماء أعلام، وأشياء مادية وأسماء حيوانات لأن المثل أحيانا ينطق به الوظيفي: المثل أحيانا يوظف الكلمة لأغراض عديدة، حيث تتعدد الدلالات، مما يتحتم على المترجم: أن يؤولما في لغة الهدف. وبالتالي على المترجم أن يبحث عن سياق يجري فيها ليتحرى 
والصعوبة في ترجمة المصطلح تختلف نوعاً ما عن الترجمة الشفهية والتفسير، لأن السؤال

الذي يطرح نفسه هل المصطلح شفاف أم غير شفاف أو مضلل، المصطلح الشفاف يكون صعب إيجاده، فنجد المضلل أسهل عند الترجمة.

معوقات ترجمة الامثال

عند ترجمة النص يواجه المترجم عدة مشاكل لأنه لا يترجم فقط الألفاظ بل يترجم

نصوصا تضمن عبارات ثقافية دينية لذا فالصعوبات أهمها تكمن في 22: 1. مشاكل المفرادات والألفاظ: إذ كانت العبارة غير معروفة عندها كيف يؤدي المترجم مهام الترجمة، خاصة المشتركات اللفظية والمتعدد اللفظي.

2. المشاكل النحوية واللغوية: تختلف اللغات عن بعضها البعض. حيث العربية بها ثلاثة أزمنة اللغة الأوروبية أزمنتها أكثر. بالإضافة إلى ذلك الضمائر والصفات تختلف فهي تمثل مشكلة أمام المترجم.

3. المشاكل الثقافية: لأن ثقافة كل مجتمع تختلف عن الأخر خاصة بين العببية والأنجليزية فهو اختلاف (غربي وشرقي) تدخل بها ثقافة دينية وإجتماعية وغير ذلك من الفروقات.

4. مشاكل في الأسلوب: أسلوب النص يمكن أن يخلق مشكلة للمترجم، مثل ظهور ترتيب للجملة بشكل غير اعتيادي و أن يكون الأسلوب مبهم.

كل ثقافة لما تركيب لساني مميز يختلف عن الثقافة الأخرى. ترى مونا بيكر ( Baker بيكر يوجد اختلاف في ثقافة اللغتين من حيث الهدف والأصل فلو حاولنا ترجمة (إذا حضر الماء بطل التيمم) نجد "التيمم" ليس موجود في الثقافة الغير الإسلامية نترجمها (حضر الأفضل بطل المفضول). ايضا كلمة "Aunt" في الأنجليزية للعمة والخالة بينما في العربية لكلتيهما كلمة منفصلة.

\section{غياب المعنى والمشاكل المتعلقة بجا}

حسب مونا بيكر الأمثال والعبارات الثابتة تحتاج إلى ترجمة بأسلوب خاص لكي نجد مكافاً لها في لغة الهدف، ولكن المشكلة تتعقد عند عدم وجود مكافئ لها في لغة الهدف لو حاولنا 
ترجمة كلمة "Oh" لأن لها مدلولات عديدة فهي تارة تدل على الضجر وتارة تدل على الدهشة. فيحتاج المترجم التمعن في النص حتى يترجمها وينقلها للغة الهدف على الوجه الصحيح. إذا حاولنا ترجمة "Excuse me" فأيضا لها عدة معاني قد تأتي بمعنى إسمح لي، ومرة تأتي عفواً وأخرى تترجم بالاعتذار فيصعب على المترجم إن لم تكن له دراية بمعناها الأصلي أن يُترجمها على الوجه الصحيح. وكذلك عندما نترجم "رمضان كريم" للأنجليزية "Ramadan is generous" فهنا المترجم لابد أن يتحقق من أن الذي يتحدث عن كرمه هو شهر الفضيلة "رمضان" أم يتحدث عن كرم شخص اسمه رمضان لا صلة له بالشهر الفضيل، ولأن العرب يستخدمون عبارة "رمضان كريم" للاعتذار عن تقديم واجب الضيافة للضيف إذا زارهم في شهر رمضان، وأيضاً تستخدم للتعبير عن الغضب في شهر رمضان.

وكذا الأمر في "البيت الحرام، البيت العتيق، بيت الله" بما أن لا مكافئ لما في لغة الهدف يصعب على المترجم أن يترجها كما هي البيت الحرام ترجمتها " The forbidden home " و "البيت العتيق" ترجمتها " The old house. " فهي تخلق مشكلة للمترجم ليس لدية خلفية عن الثقافة " The Ka'ba " الدينية ومكانة الكعبة الشريفة فيصعب عليه ترجمتها ترجمة حرفية لأن المكافئ لها المثل "اسم الله عليك" يستخدم في حالة إذا ما الطفل وقع على الأرض خطأ أو خاف من شيء ما. أو بكي من ألم، كما تستخدم أيضا عندما تقول الأم أو أي من الأقارب للتعبير عن حالة الطفل بأنه بصحة جيدة ووزن جيد ورأه أقاربة فخوفاً من أي يصيبه عين أو نظرة حسد وإيماغم بأسم الله God's name be " وقدرته، فإغم يقولون "اسم الله عليك" ويحتار المترجم لإيجاد مكافئ لما حيث يترجمها upon you بل مكافئ معادل للعبارة.

\section{استراتيجيات تستخدم لتزبمة الأمثال}

استخدم المترجمون عدة استراتيجيات لنقل المصطلح من لغة الأصل إلى الهدف يراعي

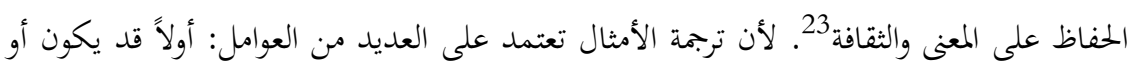


لا يكون مشاجها لها في المعنى. وعوامل أخرى قد تكون معجمية او تحدد شكل المثل فهناك إمكانية

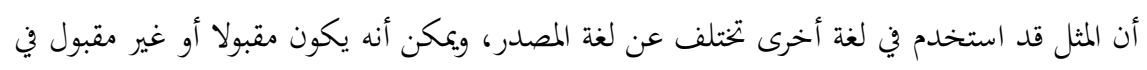
لغة الهدف، حاولنا تسليط الضوء على الاستراتيجيات تساعد في ترجمة الأمثال وهي:

التأليف

اذا جاء مثل بمضمون لا يوجد في لغة الهدف فلا تليق الترجمة الحرفية للمثل لأنه لن ينقل

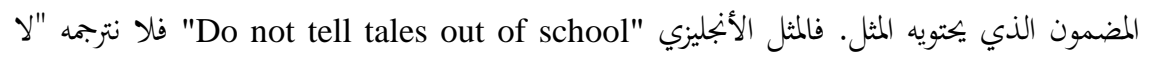
تقصص قصصا خارج المدرسة" لأنه سيخفي المضمون الثقافي في المثل ولذا نترجمها "هنا حفرنا وهنا دفنا"، نجد المثل "يصيب كبد الحقيقة" لايوجد في الأنجليزية مثال يطابقه في المعنى فلذا يلجأ المترجم

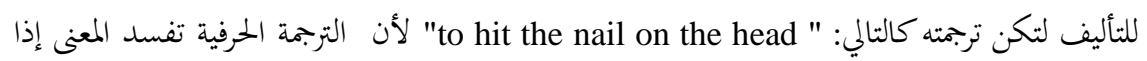
ما ترجم ب" to hit the liver of the truth". المثل العربي "ييع الماء في حارة السقايين لايوجد

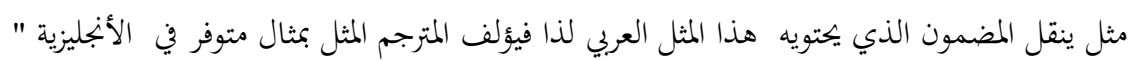
carry coals to Newcastle

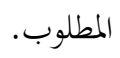

\section{زيادة الكثافة}

فالمتزجم يترجم المثل ليحوله من عام إلى خاص وبدون إضافة معلومة أخرى أي محاولة إظهار

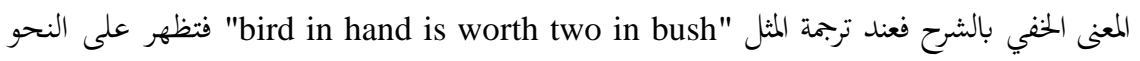
التالي "عصفور في اليد خير من عشرة على الشجرة" ان كلمة اثنان ترجمت إلى عشرة فبهذا زادت

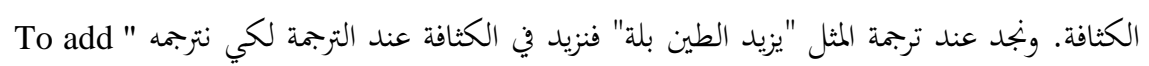
insult to injury

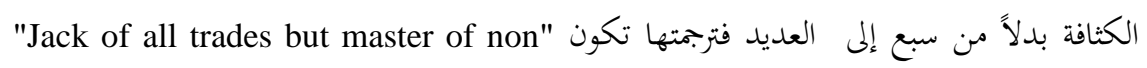
فكلمة "all" زادت الكثافة من سبع إلى الكل. 
عند ا ستخدام هذه الاستراتيجية فان المترجم يضيف محسنات من لغة الهدف عند ترجمة

المثل "A good deed is never lost" فترجمته العمل الصالح لا يضيع لكنها غير مناسبه في لغة الهدف وبإضافة المحسنات فيترجم على النحو التالي "إزرع جميلا ولو في غير مكانه " ونجد استراتيجة المسنات عند ترجمة المثل الأنجليزي "move earth and heaven" فترجمته تحريك الأرض والسماء فهي غير مناسبة في اللغة العربية لذا يلجأ المترجم ليضيف إليها محسنات لغوية ويترجمها على النحو Mice " التالي "يقيم الدنيا ويقعدها". إذا حاولنا ترجمة المثل "الفئران تجري في معدتي" بالعبارة الأنجليزية "run in my stomach

."butterflies in my stomach

إعادة الصياغة

إذا غاب المعنى أو انعدم في لغة الهدف يعيد المترجم الصياغة للجملة فمثلا " Barks is willing المثل قد ضرب لشخص اسمه باركس حالته المالية ضعيفة ويريد الزواج لذا فترجمته بالمثل العربي تكون الأنسب. ، وكذلك المثل "live from hand to mouth" فيتم إعادة صياغته المثل عند الترجمة ليكون "يحيا حياة الكفاف". نجد غياب المعنى في المثل الأنجليزي "think on one's feet" فلا يصح أن نترجمه بالعبارة "يكفر على رجل واحده فنعيد صياغة المثل ليترجم ب"سريع البديهة".

\section{التزمة بالحذف}

في بعض الحالات، يحتاج المترجم لحذف المثل لعدم احتواءه على مثل مماثل أو إذا تعذر على المترجم أن يعيد صياغته للمثل وذلك لانعدام مقابل له في لغة الهدف فإنه يلجأ لحذف المثل كاملا وإعادة صياغته بما يناسبه مع ما يراد به، وهذا عكس الزيادة التي تكون بالانتقال من الظاهر للضمني، وبالتالي يتجاوز المستوى السطحي للنص الأصلي وتحذف بعض الكلمات. فالمثل العربي

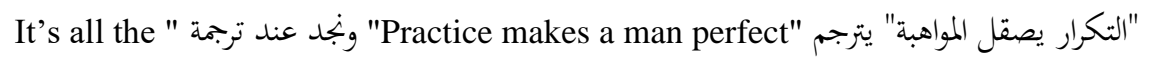
"same to you 
you " المثل العربي "حُكلي تَ أحُكلّك" أوبعبارة أخرى "شيّلني واشيّلك" فكلاهما تترجم إلى scratch my back and I'll scratch yours الترجمة بالمعنى

إذا انعدم المثل ولايوجد مرادف أو مقابل له في لغة الهدف فيتم ترجمته بمعنى معادل أو

مقارب للمثل بلغة الأصل وذلك بإيجاد مثل موازي له في لغة الهدف، ويكون ذلك في حالة الاختلاف الثقافي بين النص الأصلي والهدف لتعارض الثقافة فمثلا: " يفهمها وهي طائرة" لأن الترجمة الحرفية تكون لها "He will get it at the air" فهي لا تفهم في لغة المدف لأن لا معنى لها لذا فهي تترجم بالأنجليزية بمعنى " It's said about someone who is very intelligent ."deciphering cods عy country " عند ترجمة المثل: بلادي وإن جارت علي عزيزة. لا نستطيع ترجمتها الترجمة الحرفية East or west, " بل تكون الترجمة الأنجليزية لها "and if it Opressed me is dear to me

."home is best المثل: "أول بأول" فيترجم إلى "First with the first" فهي فقط معادل مكافئ معجمياً وليس موازية. كذلك عند ترجمة المثل المذكور في القرآن "يخفض جناحه" فتكون ترجمته الحرفية "lower "your wing "لكن الترجمة المجازية المكافئة للمعنى العربي هي: " be humble and kind" . ومن خلال الأمثلة السابقة نلاحظ أن الترجمة في هذا النوع قد تتجاوز عن السياق بطريقة ما. ومع ذلك فهي تكون مرتبطة ومشتقة من النسخة الأصلية بطريقة أو بأخرى. ولأن المصطلحات والأمثال العربية تحتوي على عناصر ثقافية وتاريخية لا تفهمها الثقافات الأخرى. كما تشكل الصعوبات في ترجمة المصطلحات والأمثال والتعبيرات المرتبطة ثقافيًا إشكالية لكل من ونسئ المتعلمين للغة الأجنبية والمترجمين. فجميع اللغات بها تعابير وأمثال ولكن ليس من السهل العثور على مكافئ لها في لغة الهدف الذي يكون مكافئاً مع المصطلح في لغة المصدر وفي المعنى والشكل. ومن أهم الصعوبات التي يواجهها المترجمون للأمثال هي القدرة على معرفة المثل وتفسيره بالشكل الصحيح وذلك من ناحية المعنى والمفهوم وكذلك لكي ينجح في العثور على معادل مكافئ للمثل. 
الترجمة عملية معقدة في حد ذاتا، ناهيك عن ترجمة المصطلحات فإن إشكالية ترجمتها أكثر صعوبة. ويُعزى ذلك إلى حقيقة أن التعبيرات الاصطلاحية لها معان غير حرفية، وعلى الرغم من ذلك فهي عملية معقدة بحد ذاتما. وبعض العوامل الأخرى قد بتعل المصطلحات ميزة، وبالتالي فإنه ليس من السهل التعامل معها. في حين أن البعض يتميز بتجميع الكلمات الفردية أو عبارات جامدة وغير مبرة والمثل جنس نثري يتميز بطابع جمالي، وأي المثل صعب حصره حيث أن الأمثال تأتي عادة موجزة وتحمل في طياتما ثقافات دينية واجتماعية وأخلاقية. وتكون الأمثال لإثبات المعنى في النفس والعبرة وهذا ما يجعل المترجم يواجه صعوبات شتى في البحث عن مكافئ لما في لغة الهدف أو إعادة صياغتها بمكافي يوصل المعنى....الخ. لذا يلجأ للتحليل والتفسير أو شرح العبارة لأن المثل يحمل رؤية خاصة بثقافة الشعب وتباين وجهات النظر يجعل المترجم يحتار ويحتاج منه جهداً مضاعفاً لينقل المعنى ويصل به إلى المعنى المرجو. لذا نجد المترجم يتجاوز المستوى اللغوي إلى لى المستوى المعادل لإيجاد مكافئ للموروث الثقافي عند ترجمة الأمثال.

النتائج

لقد لفت انتباهي أن ترجمة الأمثال من لغة إلى أخرى تحتاج لإجراء كثير من الدراسات وذلك لندرتها.

فمترجم الأمثال لابد أن يطلع وينهم بقدر كبير وواسع من ثقافة ولغة الأصل والهدف. ولابد أن يتشبع بثقافة ولغة الأصل والهدف.

لابد لمترجم الأمثال الاطلاع على المزيد من التعابير والأمثال في اللغتين كي يتمكن من

$$
\text { إيصال المعنى الصحيح والمعبر للحدث. }
$$

يجب لمترجم الأمثال الادراك للاختلافات الأسلوبية والبنيوية في لغة مصدر الأمثال ولغة

زيادة الصعوبة عند ترجمة المصطلحات والتعبيرات ذات الاختصاص من العربية للإنجليزية 


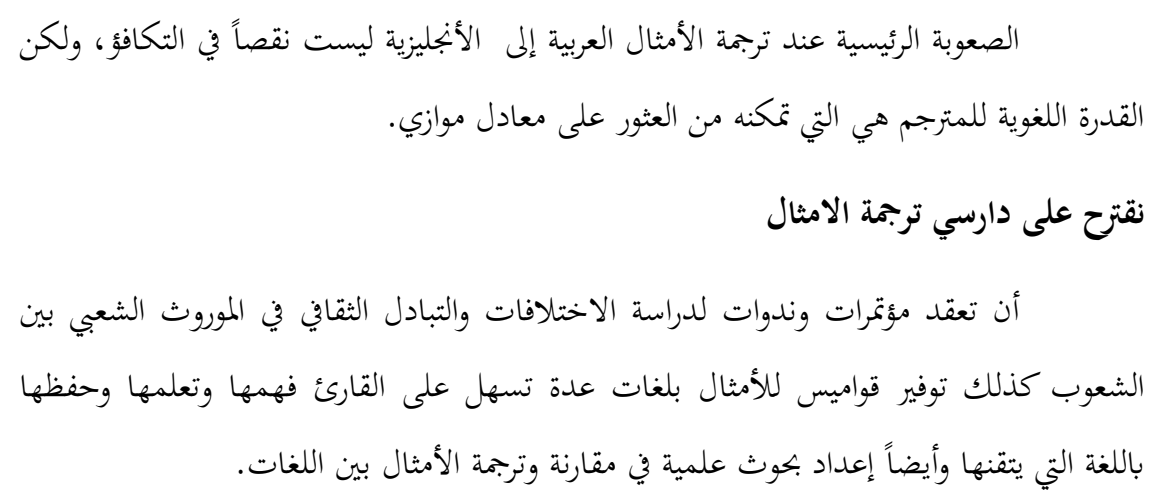

Khair al-Din Shamsi Pasha, Dictionary of Arabic Proverbs, King Faisal Center for Research and Studies, Riyadh, Volume 1, i 1, year 2002, p. 10.

$$
2{ }^{2}{ }^{2}
$$

Ibn Khaldun, Introduction, Dar Al-Fikr for Printing and Publishing, Beirut, Lebanon, 1st Edition, 2003, p. 504.

3 Newmark, 1988, Textbook of Translation. New Yourk and London: Prentice-Hall. p.104.

$$
4 \text { زمليم، الأمثال العربية القديمة. تعريب د. رمضان عبد التواب: بيروت1982. }
$$

Zelheim, ancient Arabic proverbs. Arabization d. Ramadan Abdel Tawab: Beirut.

$$
5
$$

Dr. Abd al-Majid Qatamesh, the luxurious pearl in proverbs, Dar al-Maaref edition 1971.

$$
6
$$

Ismail, Muhammad, popular proverbs and their impact on our behavior, p. 3 .

$$
7
$$

International Arabic Encyclopedia, group of researchers.

$$
8 \text { ابن عبد ربه، العقد الفريد، تعقيق أحمد أمين وآخرون، دار الكتاب العربي، بيروت، ج3 سنة 1982، ص63. }
$$

Ibn Abd Rabbo, The Unique Contract, investigated by Ahmed Amin and others, Dar alKitab al-Arabi, Beirut, vol. 31982.

$$
9 \text { 9 نور الدين السد، الأسلوبية ووتحليل الخطاب،جزء 1، ص168. }
$$

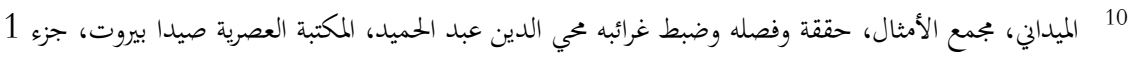

$$
\text { سنة } 2005 \text { ص6. }
$$

Al-Maidani, Complex of Proverbs, Investigating, Separating and Controlling his Oddities, Mohieddin Abdel Hamid, Modern Library, Sidon, Beirut, Part 1. 
Ibid, P105.

12

Ibid, P125.

13 Mohammed Basam Thalji, the Translation of Proverb: Obstacles and Strategies, Middle East University, Amman, Jordan, May 2015, p-1.

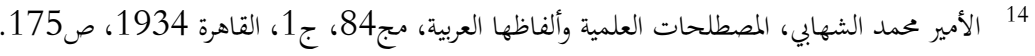

Prince Muhammad Al-Shihabi, Scientific Terms and Their Arabic Words, Volume 84, Volume 1, Cairo 1934, p. 175.

$$
15 \text { سعيد كيحل، تعليمية الترجمة دراسة تحليلية تطبيقية، عالم الكتب الحديث، الأردن ص21. }
$$

Saeed Kaihel, Teaching Translation, an Applied Analytical Study, The Modern World of Books, Jordan, p. 21.

$$
16 \text { أبن منظور، لسان العرب، المخيط، ج1، بيروت، ص316. }
$$

Ibn Manzur, Lisan Al Arab, Al Mohit, Volume 1, Beirut, p. 316.

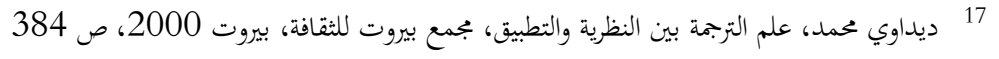

Didawi Muhammad, The Science of Translation Between Theory and Practice, Beirut Cultural Complex, Beirut 2000, p. 384.

${ }^{18}$ Mire madi, A. (1993) Theories of Translation and Interpretation. Tehran: Samt.

19 محمد الديداوي، الترجمة والتواصل، دراسات تحليلية عملية لإشكالية الاصطلاح ودور المترجم،1 1، 2000،

Muhammad Al-Didawi, Translation and Communication, Practical Analytical Studies of the Problematic Terminology and the Role of the Translator, 1, 2000, p. 52.

$$
20 \text { مازن الوعر، مشكلات الترجمة في المصطلح العربي اللساني، ص49. }
$$

Mazen Al-Waer, Translation Problems in the Arabic Linguistic Terminology, p.49.

$$
21
$$

"The lizard" speaks in the Proverbs Complex for the field.

$$
22 \text { محمد شهين نظريات الترجمة وتطبيقاتحا في تدريس الترجمة، مكتبة دار الثقافة ، عمان 1998، ص83. }
$$

Muhammad Shaheen, translation theories and their applications in teaching translation, Dar Al-Thaqafa Library, Amman 1998, p. 83.

${ }^{23}$ Hatim, B. and Mayson, I (1990) Discourse and the translator. London and New Yorkp38. 